\title{
Modulation of nuclear factor- $\kappa B$-mediated pro-inflammatory response is associated with exogenous administration of bone marrow-derived mesenchymal stem cells for treatment of experimental colitis
}

\author{
DONGMEI ZUO*, QING TANG* , HENG FAN, ZHEXING SHOU, XINGXING LIU, DAN CAO and ZHOU ZOU \\ Department of Integrated Traditional Chinese and Western Medicine, Union Hospital, Tongji Medical College of \\ Huazhong University of Science and Technology, Wuhan, Hubei 430022, P.R. China
}

Received February 10, 2014; Accepted November 3, 2014

DOI: $10.3892 / \mathrm{mmr} .2014 .3038$

\begin{abstract}
Mesenchymal stem cells (MSCs) inhibit the immune response in vitro and prevent the induction of disease in certain experimental models. As a result, MSC-mediated therapy is a rapidly growing field of research. However, the efficacy of MSCs in the treatment of inflammatory bowel disease (IBD) has remained to be determined. In the present study, rats with 2,4,6-trinitrobenzene sulfonic acid (TNBS)-induced colitis were injected with prepared MSCs $\left(1 \times 10^{6}\right)$ into the tail vein. Two weeks following intravenous MSC administration, the concentration of tumor necrosis factor- $\alpha(\mathrm{TNF}-\alpha)$ in the serum was measured by an ELISA. The protein expression of nuclear factor $-\kappa \mathrm{B}(\mathrm{NF}-\kappa \mathrm{Bp} 65)$ in the colonic mucosa was assessed by western blot analysis. mRNA expression of TNF- $\alpha$ and NF- $\kappa$ Bp65 was determined by reverse-transcription quantitative polymerase chain reaction. MSCs were shown to exert an immunomodulatory effect on TNBS-induced colitis and may be of use in the treatment of IBD. In addition, modulation of the NF- $\kappa \mathrm{B}-$ mediated pro-inflammatory response may contribute to the underlying mechanism by which MSCs ameliorate the clinical and histological changes associated with IBD.
\end{abstract}

Correspondence to: Professor Heng Fan or Dr Zhexing Shou, Department of Integrated Traditional Chinese and Western Medicine, Union Hospital, Tongji Medical College of Huazhong University of Science and Technology, 1277 Jiefang Avenue, Wuhan, Hubei 430022, P.R. China

E-mail: fanheng009@aliyun.com

E-mail: 36747601@qq.com

*Contributed equally

Key words: bone marrow mesenchymal stem cells, nuclear factor- $\kappa \mathrm{B}$, tumor necrosis factor $\alpha$, inflammatory bowel disease

\section{Introduction}

Crohn's disease (CD) and ulcerative colitis (UC) are the two most common forms of inflammatory bowel disease (IBD). They are chronic inflammatory disorders, which may be progressive or relapsing, and are characterized by a range of symptoms, including abdominal pain, severe diarrhoea, rectal bleeding and wasting. The pathological findings in IBD are correlated with a number of factors, including genetic predisposition, environmental factors, gut dysbiosis and an inadequate immune response (1-3). Retrospective studies have shown that the balance between pro- and anti-inflammatory cytokines is critical in maintaining normal gut homeostasis in the colonic mucosa. A disturbance of the cytokine profile, in particular, pro-inflammatory cytokine overexpression, has been reported in IBD $(4,5)$. The present treatment for IBD comprises a number of approaches, including the rapid induction of clinical remission, steroid-free maintenance of clinical remission, mucosal healing and improvements in quality of life. Other anti-tumor necrosis factor (TNF) agents and novel biological therapies have been developed and introduced into clinical trials for IBD $(6,7)$. Despite recent advances in the clinical management of IBD, the long-term efficacy of these agents remains to be determined.

Mesenchymal stem cells (MSCs) are easily isolated from a number of tissue sources, including bone marrow (BM), fat, the umbilical cord and other tissues with the ability to differentiate into multiple cell lineages, including adipocytes, chondrocytes and osteocytes. They are a promising tool for use in cell therapies (8). MSCs are able to directly differentiate into multiple cell lineages and also indirectly exhibit a range of immunomodulatory functions through the secretion of proteins and cytokines. As BM-derived MSCs (BM-MSCs) are easily isolated from adult sources, may be cultured in vitro, display low expression of HLA and costimulatory molecules, and are relatively free from ethical controversy, they have been used in a number of preclinical and clinical studies $(9,10)$. An early study into MSC immunobiology showed that MSCs acquire immunosuppressive or immunostimulating properties within a typical inflammatory environment (11). More recently, MSCs were shown to exhibit immunomodulatory functions in innate 
and adaptive immune responses. Other findings have demonstrated that MSC transplantation therapies may be applied to graft versus host disease and CD $(12,13)$.

Increasing evidence indicated that a number of the common immunological responses present in IBD are mediated by cytokines. MSCs are also known to regulate the immune function by modulating the secretion of pro-inflammatory cytokines and chemokines in inflamed tissues. However, the effect of exogenously administered MSCs on IBD remains to be elucidated. In the present study, the effect of exogenously administered BM-MSCs in a 2,4,6-trinitrobenzenesulfonic acid (TNBS)-induced rat model of colitis was investigated in addition to the possible interactions between MSCs and the pro-inflammatory response in mediating this process.

\section{Materials and methods}

Animals. Female Sprague Dawley rats (age, 6-8 weeks), which were specific pathogen-free and weighed 200-250 g, were purchased from the Experiment Animal Center of Centers for Disease Control (Hubei, China). Rats were randomly assigned to each group ( $\mathrm{n}=6$ per group). Then were given ad libitum access to water and a standard diet; rats were kept in a temperature controlled enviroment $\left(20-22^{\circ} \mathrm{C}\right)$, with a humidity of $\sim 52 \%$ and a 12-h light/dark cycle. The present study was approved by the ethics committee of Tongji Medical College, Huazhong University of Science and Technology (Wuhan, China) and the experimental protocol was approved by the Experimental Animal Center of Tongji Medical College, Huazhong University of Science and Technology.

Isolation, culture and characterization of BM-MSCs. The isolation and culture of BM-MSCs was conducted as previously described (14). Four-week-old rats were sacrificed by cervical dislocation. Rats were immersed in $75 \%$ ethanol for $5 \mathrm{~min}$, following which the bone marrow was isolated bilaterally from femurs and tibias. BM mononuclear cells were isolated by density gradient centrifugation for $5 \mathrm{~min}$ at $350 \mathrm{x}$ g. Cells were plated in a plastic tissue culture flask (Corning Inc., Corning, NY, USA) and cultured in a low-glucose complete cell culture medium consisting of a minimum essential medium (a-MEM; GIBCO, Life Technologies, Grand Island, NY, USA) with $10 \%$ fetal bovine serum (FBS; GE Healthcare, Life Sciences, Logan, UT, USA). Nonadherent cells were removed by replacing the medium at $48 \mathrm{~h}$ and every 3-4 days thereafter. All cultures were maintained at $37^{\circ} \mathrm{C}$ in a $5 \% \mathrm{CO}_{2}$ atmosphere. Over the course of 1-2 weeks, adherent cells were collected using $0.25 \%$ trypsin solution (GIBCO). Cells were passaged once they reached $\sim 80 \%$ confluency. Third-passage cells were used in subsequent experiments. To evaluate the surface marker phenotype of the cultured MSCs, cells were trypsinized and incubated with the following fluorescent anti-rat monoclonal antibodies for $30 \mathrm{~min}$ at room temperature: Anti-CD29-phycoerythrin (PE)-Cy7; anti-CD90-AlexaFluor ${ }^{\circledR} 488$; anti-CD45-PE; and anti-CD11b-AlexaFluor ${ }^{\circledR} 647$ (BioLegend Inc., San Diego, CA, USA). Cells were washed twice with phosphate-buffered saline (PBS; Biosciences Co., Ltd., Wuhan, China), and then resuspended in PBS. Detection of PE-Cy7/AlexaFluor488/PE and AlexaFluor647 labeling was conducted using flow cytometry (FACSCalibur; Becton-Dickinson, Franklin Lakes, NJ, USA).

Transduction of BM-MSCs with green fluorescent protein (GFP). Third-passage BM-MSCs at $\sim 40 \%$ confluence were seeded in fibronectin-coated six-well plates (Corning Inc.). At $24 \mathrm{~h}$ following plating, the medium containing 10\% FBS was removed. Transduction was conducted at a multiplicity of infection (MOI) of 15 units, according to the manufacturer's instructions. Cells were added to the recombinant replication-defective lentivirus carrying GFP (LV-GFP; Genechem Co., Ltd., Shanghai, China) supernatant, containing $5 \mu \mathrm{g} / \mathrm{ml}$ polybrene (Genechem Co., Ltd.), to obtain a final volume of $3 \mathrm{ml}$. Following incubation with LV-GFP for $2 \mathrm{~h}$, the transduction medium was replaced with a fresh culture medium containing $10 \%$ FBS. An additional transduction was conducted at $48 \mathrm{~h}$. The expression of the GFP transgene in the BM-MSCs was observed using fluorescence microscopy (GpJ9-TS100-F; Nikon, Co., Tokyo, Japan). BM-MSCs were then trypsinized for five minutes and used in the subsequent experiments.

Induction of experimental colitis and treatment. In the present study, TNBS (Sigma-Aldrich, St. Louis, MO, USA) was used to induce an experimental colitis as described previously (15). On days 0, 3 and 7, GFP-transduced BM-MSCs at a dose of $1 \times 10^{6}$ cells in $0.3 \mathrm{ml}$ PBS were injected into the tail vein of the rats with TNBS-induced colitis. In the control experiments, animals received $0.3 \mathrm{ml}$ PBS without BM-MSCs and followed an otherwise identical protocol. The disease activity index (DAI) was recorded as a combination of weight loss, stool consistency and bleeding as previously described (16). Scores were assigned according to the following criteria: (1) Weight loss $(0,<1 \% ; 1,1-5 \% ; 2,5-10 \% ; 3,10-15 \%$; and 4 , $>15 \%$ ); (2) stool consistency (0, normal; 2 , soft stools; and 4, liquid stools); and ( 3 ) rectal bleeding ( 0 , negative; 2 , positive; and 4 , serious bleeding). On day 15 , mice were sacrificed and blood was collected by ventral aortic puncture for the analysis of serum inflammatory cytokine levels. In addition, the entire colon was excised and colon tissue samples were harvested for histological examination and evaluation of the mRNA expression of inflammatory cytokines in the intestinal mucosa.

Histological examination. Colon samples were fixed in $4 \%$ paraformaldehyde, embedded in paraffin (Rutgers Co., Ltd.), and cut into sections $(4 \mu \mathrm{m})$ prior to staining with hematoxylin and eosin (Rutgers Co., Ltd.). Histological evaluation was completed semi-quantitatively according to the scale described previously (17). The parameters that were evaluated included the extent of mucosal injury, leukocyte infiltration, crypt abscesses and loss of goblet cells. Each of these parameters was graded on a 0-3 scale according to the following criteria: 0 , none; 1 , slight; 2 , moderate; 3 , severe. The final histological score was defined as the sum of the scores of these parameters.

Tracing of BM-MSCs by GFP labeling. BM-MSCs were transduced with LV-GFP as described previously (18) in order to trace the infused BM-MSCs in vitro. Colonic tissue 
samples, excised from the inflamed and the non-inflamed regions of the colon, were embedded in an optimum cutting temperature compound (Sakura Finetechnical Co., Ltd., Tokyo, Japan), and frozen in dry ice. One of these sections was used to detect GFP-positive cells via fluorescence confocal microscopy (E600; Nikon Co.) and the other was stained with an antibody against GFP (1:600; EMD Millipore, Billerica, MA, USA) and visualized using a fluorescein isothiocyanate (FITC)-conjugated secondary antibody (Santa Cruz Biotechnology, Inc., Dallas, TX, USA). In addition, further samples were prepared in order to analyze the expression of the GFP protein by western blotting.

Detection of serum pro-inflammatory cytokines. Blood samples were centrifuged at $1,000 \mathrm{x}$ g for $15 \mathrm{~min}$ and the sera were stored at $-80^{\circ} \mathrm{C}$ prior to the evaluation of cytokine levels. TNF- $\alpha$ concentration in the sera was determined using a rat TNF- $\alpha$ enzyme-linked immunosorbent assay (ELISA) kit (BioSource, Inc., Camarillo, CA, USA) according to the manufacturer's instructions.

Western blot analysis. Total protein was isolated and quantified using a bicinchoninic acid protein assay kit (Pierce-Perbio Science, Tattenhall, UK). The isolation of nuclear proteins was performed using NE-PER ${ }^{\circledR}$ nuclear and cytoplasmic extraction reagents (Thermo Fisher Scientific, Waltham, MA, USA). Ice-cold nuclear extraction buffer $(25 \mu \mathrm{l})$ was added and the mixture was incubated for 30 min with intermittent mixing. Extracts were centrifuged at $1,500 \mathrm{x} g$ for $15 \mathrm{mins}$ and the supernatant (consisting of the nuclear extracts) was stored at $-80^{\circ} \mathrm{C}$ prior to use. The primary antibody against $\mathrm{NF}-\kappa \mathrm{B}$ p 65 was used at a dilution of 1:1,000. For evaluation of the levels of the control proteins, anti-ACTION and anti-HISTON antibodies were used at a dilution of 1:10,000. Protein extracts were resolved by $0.1 \%$ SDS-PAGE electrophoresis (Beyotime Co., Ltd., Shanghai, China) and transferred onto polyvinylidene difluoride membranes (Rutgers Co., Ltd.), which were blocked with $5 \%$ bovine serum albumin and incubated with the relevant antibody.

mRNA expression of TNF- $\alpha$ and NF- $\kappa B p 65$ in the colon. Colonic segments were frozen in liquid nitrogen at $-80^{\circ} \mathrm{C}$ prior to use. RNA was isolated from colonic tissues using TRIzol $^{\circledR}$ reagent (Invitrogen Life Technologies, Carlsbad, CA, USA) according to the manufacturer's instructions. cDNA was synthesized from $0.5 \mu \mathrm{g}$ total RNA using a reverse transcription (RT) kit (Toyobo Co., Ltd., Osaka, Japan) according to the manufacturer's instructions. Following this step, a quantitative polymerase chain reaction (qPCR) using the SYBR-Green I Realtime PCR Master mix (Takara Bio, Inc., Otsu, Japan) with a final volume of $20 \mu \mathrm{l}$, was conducted using the ABI PRISM 7900 sequence detector system (Applied Biosystems, Life Technologies, Foster City, CA, USA). Primers (Takara Bio, Inc., Dalian, China) were designed according to data from Gen-Bank and the sequences of primers for the qPCR experiments were as follows: Forward, 5'-CCGTCTCCTACCAGACCAAGG-3' and reverse, 5'-CTGGAAGACCCCTCCCAGATAG-3' for TNF- $\alpha$; forward, 5'-CTTCTCGGAGTCCCTCACTG-3' and reverse, 5'-CCAATAGCAGCTGGAAAAGC-3' for $\mathrm{NF}-\kappa \mathrm{B}$; and forward, 5'-GGGGCTCTCTGCTCCTCCCTG-3' and reverse, 5'-CGGCCAAATCCGTTCACACCG-3' for GAPDH. The relative gene expression levels (the amount of target mRNA, normalized to that of the endogenous and control genes) was calculated using the comparative $\mathrm{Ct}$ method via the formula $2^{-\Delta \Delta \mathrm{Ct}}$.

Statistical analysis. Values are presented as the mean \pm standard deviation. The un-paired t-test and the Mann-Whitney $\mathrm{U}$ test were used for parametric and non-parametric analyses between two groups, which was assessed using SPSS 18.0 software (IBM, Armonk, NY, USA) and Microsoft EXCEL 2003 (Microsoft Corp., Redmond, WA, USA). P<0.05 was considered to indicate a statistically significant difference between values.

\section{Results}

Characterization of BM-MSCs. Flow cytometric analysis confirmed that the BM-MSCs were positive for CD29 and CD90, but that they stained negative for the hematopoietic surface markers CD45 and CD11b (Fig. 1).

General conditions. All TNBS-treated rats developed looser, bloody and purulent stools, and exhibited a marked weight loss following administration of this compound. By contrast, rats that were not treated with TNBS did not display any of these symptoms and gained weight over time. Following infusion of BM-MSCs, the DAI score of the TNBS-treated rats gradually decreased and the symptoms were significantly alleviated in comparison with those in the PBS-treated control group (Fig. 2).

Histological findings. On microscopic examination, rats in the BM-MSC group had a relatively intact structure of their colonic mucosa, with more organized mucosal glands, more abundant goblet cells, a milder degree of congestion and edema, and less infiltration of inflammatory cells in the mucosa and sub-mucosa compared with the PBS-treated control group (Fig. 3). The histological score, which was defined as the sum of scores of the parameters outlined in the materials and methods section, was significantly reduced in the BM-MSC-treated group compared with that in the PBS-treated group (Fig. 4).

Localization of infused BM-MSCs in the colon. In vitro, a large proportion of GFP-labeled BM-MSCs were observed (Fig. 5B). GFP fluorescence was not detected using a confocal microscope in vivo. Distinct GFP-positive cells were observed when a GFP antibody and FITC-conjugated secondary antibody were used as described in the materials and methods section (Fig. 5C). Furthermore, western blotting revealed a high expression of GFP in the colonic tissue was observed in the GFP-labeled BM-MSC group (Fig. 6). By contrast, no immunoreactivity was detected in the PBS-treated control group or the normal control group.

Concentration of serum TNF- $\alpha$. The effect of administration of BM-MSCs on the serum levels of TNF- $\alpha$ was 

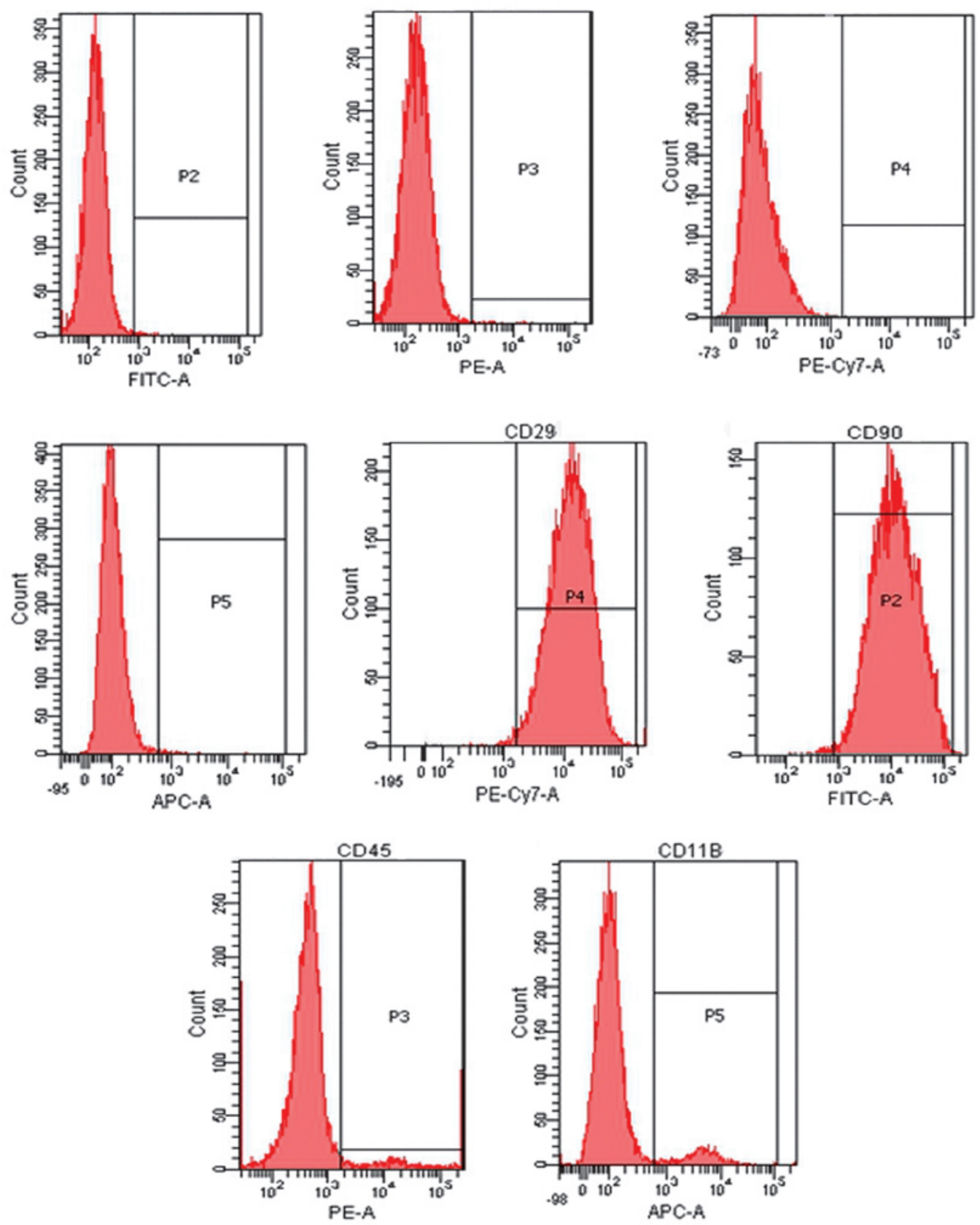

Figure 1.Flow cytometric analysis of cell-surface antigens of BM-MSCs. Cells were stained with monoclonal antibodies conjugated to PE-Cy7/AlexaFluor488/PE and AlexaFluor647. BM-MSCs expressed surface antigens for CD29 and CD90, but not for CD45 or CD11b. BM-MSCs, bone marrow-derived mesenchymal stem cells; PE, phycoerythrin; FITC, fluorescein isothiocyanate.

evaluated using an ELISA. A significant increase in TNF- $\alpha$ levels was observed in the PBS-treated group of rats with TNBS-induced colitis. Following treatment with BM-MSCs, the concentration of TNF- $\alpha$ was significantly reduced in the treatment group compared with that in the PBS control group (Fig. 7).

Protein expression of $N F-\kappa B p 65$ in colonic mucosa. The total protein and nucleoprotein expression of NF- $\mathrm{kBp} 65$ in the inflamed colon was investigated. Western blotting showed that total protein and nucleoprotein were strongly expressed in the PBS-treated colitis group. Systemic administration of BM-MSCs markedly downregulated the expression of p65 in nucleoprotein. However, no significant difference in the expression of $\mathrm{p} 65$ in total protein was identified between the MSC-treated and the PBS-treated groups (Fig. 8).

mRNA expression of TNF- $\alpha$ and NF- $\kappa B p 65$ in the colonic тисов $a$. mRNA expression of TNF- $\alpha$ and NF-кBp65 in the colonic mucosa was significantly increased in the PBS-treated group compared with that in the control group. As shown in Fig. 9, treatment with BM-MSCs resulted in a reduction of the expression of TNF- $\alpha$ and NF- $\kappa B p 65$.

\section{Discussion}

The mechanisms by which MSCs exert their reparative benefits have remained elusive. However, numerous studies 


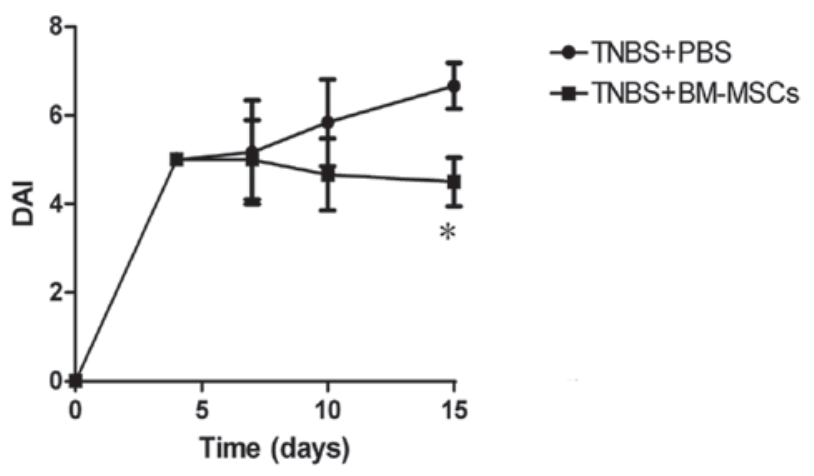

Figure 2. Transplantation of BM-MSCs protects against TNBS-induced colitis. The DAI score consisting of body weight, stool consistency and rectal bleeding is shown. Data are presented as the mean \pm standard error of the mean $(n=6)$ of three separate experiments. ${ }^{*} \mathrm{P}<0.05$ compared with the PBS-treated group. BM-MC-MSCs, bone marrow-derived mesenchymal stem cells; TNBS, 2,4,6-trinitrobenzene sulfonic acid; DAI, disease activity index; PBS, phosphate-buffered saline.
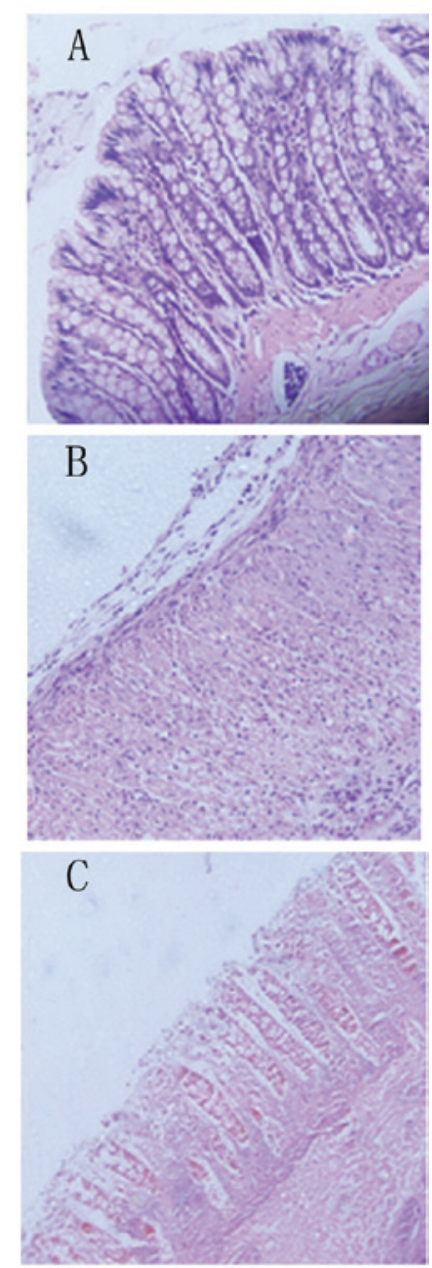

Figure 3. Histological analysis following BM-MSC therapy in experimental colitis. Colon specimens were sectioned and stained with hematoxylin and eosin. (A) Control group; (B) phosphate-buffered saline-treated group and (C) BM-MSC-treated group (magnification, x20). BM-MSCs, bone marrow-derived mesenchymal stem cells.

show that BM-MSCs are present in areas of inflammation and promote tissue repair by differentiation into a range of cell lineages. Recently, MSCs have been demonstrated to possess

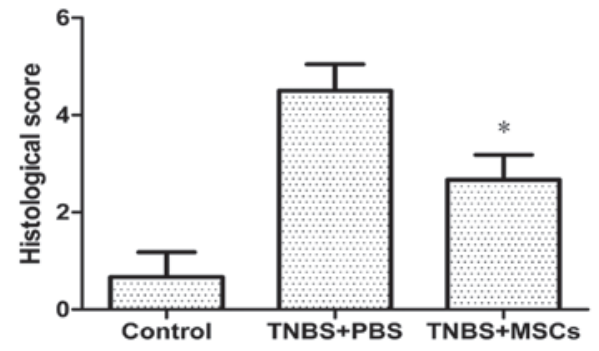

Figure 4. Effects of BM-MSC administration on histological scores. Histological severity score was lower in the BM-MSC-treated group compared with the PBS-treated group. Data are presented as the mean \pm standard error of the mean $(n=6)$ from three separate experiments. ${ }^{*} \mathrm{P}<0.05$ compared with the PBS-treated and control groups. BM-MSC, bone marrow-derived mesenchymal stem cell; PBS, phosphate-buffered saline; TNBS, 2,4,6-trinitrobenzene sulfonic acid.
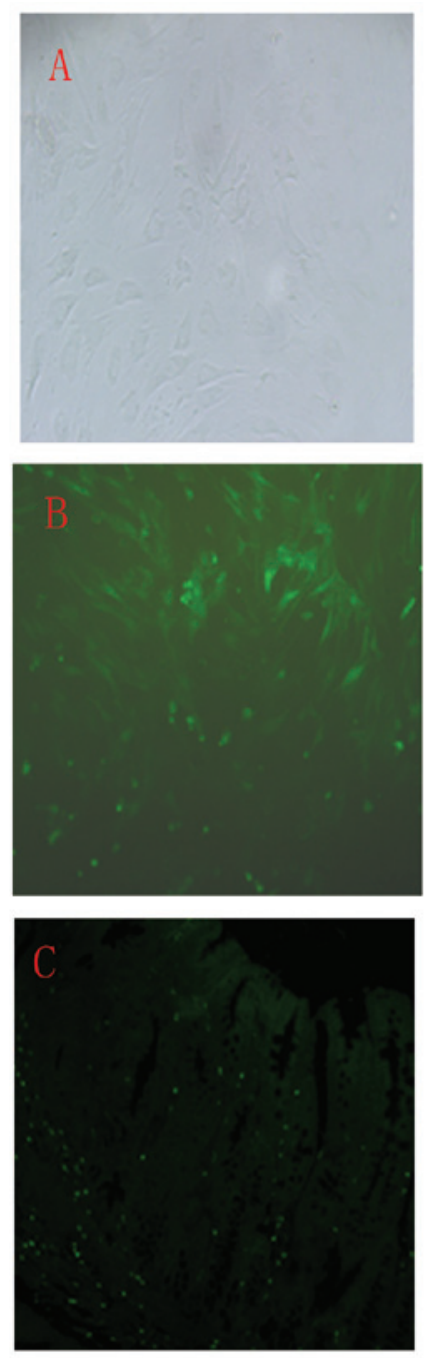

Figure 5. Localization of administered BM-MSCs. (A) Recombinant replication-defective LV-GFP-labeled BM-MSCs under a common microscope; (B) in vitro LV-GFP-labeled BM-MSCs; and (C) in vivo exogenously administered BM-MSCs were observed in the inflamed colon (magnification, $\mathrm{x} 20$ ). LV-GFP, lentivirus carrying green fluorescent protein; BM-MSCs, bone marrow-derived mesenchymal stem cells.

immunomodulatory properties, including the suppression of T-cell proliferation, influence on dendritic cell maturation and function and the suppression of B-cell proliferation and terminal 


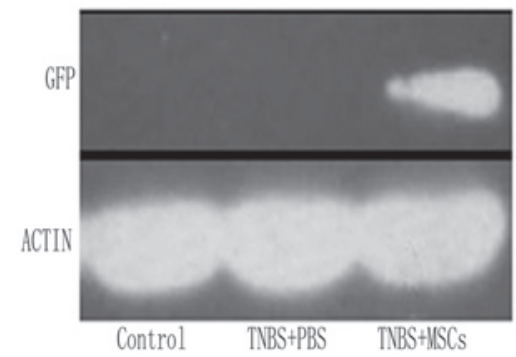

Figure 6. Western blot analysis of GFP. GFP, green fluorescent protein; TNBS, 2,4,6-trinitrobenzene sulfonic acid; PBS, phosphate-buffered saline; MSCs, mesenchymal stem cells.

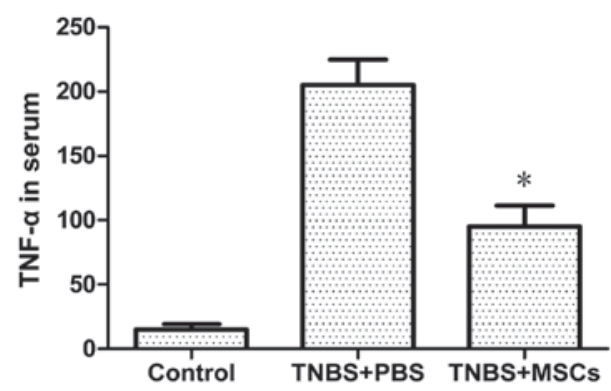

Figure 7. Effect of BM-MSCs on the concentration of TNF- $\alpha$ in serum. Data are expressed as the mean \pm standard error of the mean $(n=6)$ from three separate experiments. ${ }^{*} \mathrm{P}<0.05$ compared with the PBS-treated and control groups. BM-MSCs, bone marrow-derived stem cells; TNF- $\alpha$, tumor necrosis factor- $\alpha$; TNBS, 2,4,6-trinitrobenzene sulfonic acid; PBS, phosphate-buffered saline.

differentiation, as well as the immune modulation of other immune cells, including NK cells and macrophages (19-21). The majority of in vitro studies have demonstrated that MSCs limit T-cell expansion by impairing interferon- $\gamma$ and TNF- $\alpha$ production in addition to increasing the production of IL-10 (22). Recently, Kazunari et al (23) reported that in the systemic injection of ex vivo-cultured BM-MSCs, these cells accumulated exclusively in the region of the inflamed rectum and localized in the lamina propria, in particular at the base of the crypts. Another study demonstrated that MSCs may treat dextran sulfate sodium-induced colitis via an interaction with immune mediators, including TNF- $\alpha$, interleukin-1 $\beta$ and cyclooxygenase-2 (24).

Members of the TNF protein superfamily exist in either a membrane-bound or soluble form. The family contains 18 type 2 proteins, and the receptors for these ligands are type 1 transmembrane proteins $(25,26)$. Binding of TNF-like ligands to their receptors, including the TNF/TNF-receptor protein superfamilies, triggers the activation of intracellular pathways, which are involved in numerous components of the immune response, including direct involvement in cell proliferation, differentiation and survival (25-27). TNF- $\alpha$ is one of the primary cytokines involved in the pathogenesis of IBD (28). A number of possible mechanisms underlying its protective effects in this disease have been postulated. One factor may be that TNF- $\alpha$ effects the apoptotic elimination of effector immunocytes in the lamina propria. A second possible mechanism is the upregulation of endogenous corticosteroids by TNF- $\alpha$. A third possible factor is that TNF- $\alpha$ is able to maintain the integrity of the epithelial barrier (29-31).
A

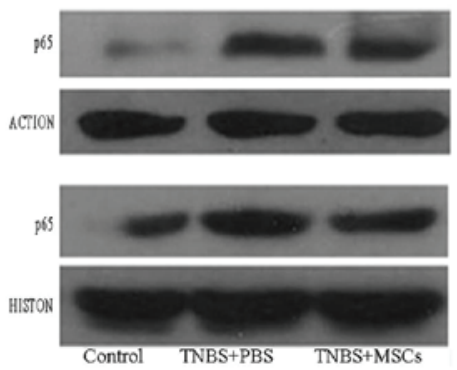

B
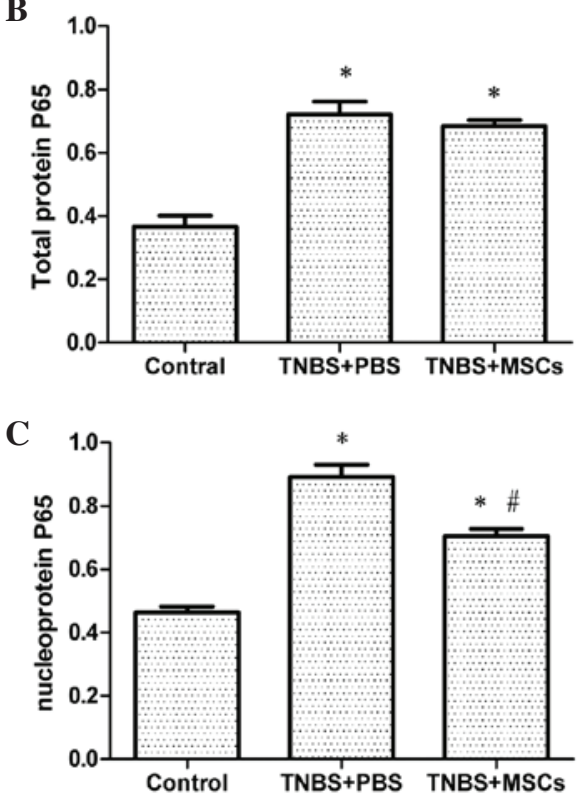

Figure 8. (A) Western blot analysis of protein expression of NF- $\mathrm{KBp} 65$ in colonic mucosa. (B) Quantification of p65 expression in total protein. (C) Expression of p65 in nucleoprotein. Data are presented as the mean \pm standard error of the mean $(n=6)$ from there separate experiments. ${ }^{*} \mathrm{P}<0.05$ compared with control group and ${ }^{\#} \mathrm{P}<0.05$ compared with PBS-treated group. NF-кBp65, nuclear factor- $\kappa$ B65; TNBS, 2,4,6-trinitrobenzene sulfonic acid; PBS, phosphate-buffered saline; MSCs, mesenchymal stem cells.

A

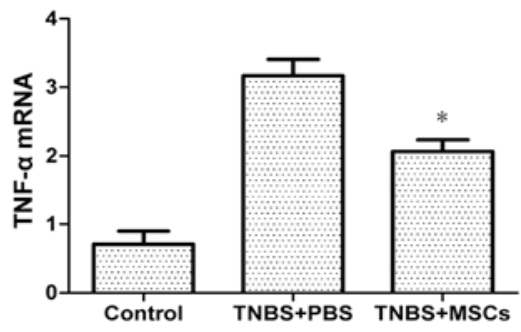

B

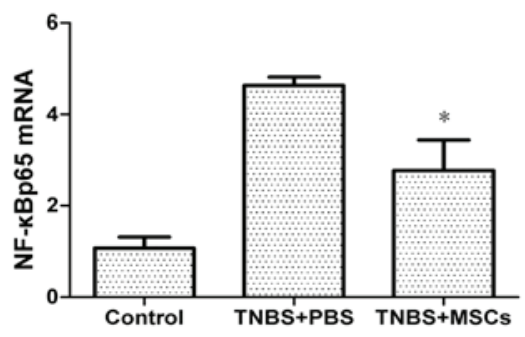

Figure 9. Colonic mucosal mRNA expression of (A) TNF- $\alpha$ and (B) NF- $\kappa$ Bp65. Data are presented as the mean \pm standard error of the mean $(n=6)$ from three separate experiments). ${ }^{*} \mathrm{P}<0.05$ compared with PBS-treated group. TNF- $\alpha$, tumor necrosis factor- $\alpha$; NF- $\kappa$ Bp 65 , nuclear factor- $\kappa \mathrm{Bp} 65$; TNBS, 2,4,6-trinitrobenzene sulfonic acid; PBS, phsophate-buffered saline; MSCs, mesenchymal stem cells. 
Subsequent research has shown that the serum levels of TNF- $\alpha$ are negatively correlated with the clinical activity of UC and CD, which may indicate the use of anti-TNF therapies for the treatment of Crohn's Disease $(32,33)$. To the best of our knowledge, there is currently no study reporting the administration of anti-TNF agents as a first-line therapy in UC, but a recent study suggested that biological therapies may have potential for use as first-line treatments in the future. In the present study, serum TNF- $\alpha$ levels were assayed by ELISA and mRNA expression of TNF- $\alpha$ in the colonic mucosa was evaluated by RT-qPCR. The data showed that following the administration of BM-MSCs, the concentration of TNF- $\alpha$ in the serum and its mRNA expression in the colonic mucosa decreased significantly compared with that in the PBS control group.

There are five members of the NF- $\kappa \mathrm{B}$ family: RelA (p65), RelB, C-Rel, p105 (NF-kB1; a precursor of p50) and p100 (NF-kB2; a precursor of p52) (34). The NF- $\kappa B$ family is a family of transcription factors and has been hypothesized to be involved in tumorigenesis, inflammation and cellular processes, including cell proliferation and apoptosis (35). Under unstimulated conditions, NF- $\kappa \mathrm{B}$ is maintained in the cytoplasm in an inactive form by interaction with a family of inhibitor proteins, termed $\mathrm{I} \kappa \mathrm{B}$ proteins. $\mathrm{NF}-\kappa \mathrm{B}$ activation occurs in response to various stimuli, when the rapid phosphorylation of I $\mathrm{B}$ leads to its degradation by the proteasome pathway, resulting in the migration of $\mathrm{NF}-\kappa \mathrm{B}$ into the nucleus $(36,37)$. NF- $\kappa \mathrm{B}$ is one of the primary factors involved in the formation of the molecular network, which can lead to various changes in cellular function that are associated with IBD. For example, IL-1, TNF- $\alpha$, IL-12 and IL-23 are NF- $\mathrm{NB}$-dependent pro-inflammatory mediators and are known to be upregulated in patients with $\operatorname{IBD}(38,39)$. The role of NF- $\kappa \mathrm{B}$ in the transcriptional control of a number of inflammatory genes, including cytokines, chemokines, growth factors and leukocyte adhesion molecules, as well as the involvement of ROS, led to the concept of NF- $\kappa \mathrm{B}$ as a therapeutic target in numerous disorders. It has been reported that antibodies targeting NF- $\mathrm{NB}$ and pro-inflammatory cytokines, including TNF- $\alpha$ and IL-6 and their signaling pathways, were effective in ameliorating the inflammation-associated intestinal damage in patients with IBD $(40,41)$. In the present study, the protein expression of NF- $\mathrm{NBp} 65$ in the inflamed colon was assessed by western blot analysis. Systemic administration of BM-MSCs markedly downregulated the expression of nucleoprotein but not that of the total protein. However, data obtained from RT-qPCR suggested that the BM-MSCs downregulated mRNA expression of NF- $\kappa$ Bp 65 in the colonic mucosa. This information suggested that BM-MSCs may affect TNBS-induced colitis via modulation of the NF- $\kappa \mathrm{B}-$ mediated pro-inflammatory response.

In conclusion, BM-MSCs may attenuate TNBS-induced colitis in rats. Increased body weight and a marked histological improvement were observed in the BM-MSC-treated group compared with those in the PBS-treated control group. Therefore, the present study demonstrated that modulation of the $\mathrm{NF}-\kappa \mathrm{B}$-mediated pro-inflammatory response was associated with exogenous administration of BM-MSCs in the treatment of experimental colitis. Future studies should focus on investigating the effect of MSCs on the NF- $\kappa \mathrm{B}$-mediated pro-inflammatory signaling pathway.

\section{Acknowledgements}

This study was supported by the National Natural Science Foundation of China (grant nos. 81273906 and 81102690).

\section{References}

1. Schirbel A and Fiocchi C: Inflammatory bowel disease: Established and evolving considerations on its etiopathogenesis and therapy. J Dig Dis 11: 266-276, 2010.

2. Baumgart DC and Carding SR: Inflammatory bowel disease: cause and immunobiology. Lancet 369: 1627-1640, 2007.

3. Hisamatsu T, Kanai T, Mikami Y, et al: Immune aspects of the pathogenesis of inflammatory bowel disease. Pharmacol Ther 137: 283-297, 2013.

4. Múzes G, Molnár B, Tulassay Z and Sipos F: Changes of the cytokine profile in inflammatory bowel diseases. World J Gastroenterol 18: 5848-5861, 2012.

5. Xavier RJ and Podolsky DK: Unravelling the pathogenesis of inflammatory bowel disease. Nature 448: 427-434, 2007.

6. de Zoeten E and Mamula P: What are the guidelines for using biologics in pediatric patients? Inflamm Bowel Dis 14 (Suppl 2): S259-S261, 2008.

7. Taba Taba Vakili S, Taher M and Ebrahimi Daryani N: Update on the management of ulcerative colitis. Acta Med Iran 50: 363-372, 2012.

8. Parekkadan B and Milwid JM: Mesenchymal stem cells as therapeutics. Annu Rev Biomed Eng 12: 87-117, 2010.

9. Meyerrose T, Olson S, Pontow S, et al: Mesenchymal stem cells for the sustained in vivo delivery of bioactive factors. Adv Drug Deliv Rev 62: 1167-1174, 2010.

10. Le Blanc K, Tammik C, Rosendahl K, Zetterberg E and Ringdén O: HLA expression and immunologic properties of differentiated and undifferentiated mesenchymal stem cells. Exp Hematol 31: 890-896, 2003.

11. Le Blanc K, Tammik L, Sundberg B, Haynesworth SE and Ringdén $\mathrm{O}$ : Mesenchymal stem cells inhibit and stimulate mixed lymphocyte cultures and mitogenic responses independently of the major histocompatibility complex. Scand J Immunol 57: 11-20, 2003.

12. Dalal J, Gandy K and Domen J: Role of mesenchymal stem cell therapy in Crohn's disease. Pediatr Res 71: 445-451, 2012.

13. Ringdén O, Uzunel M, Rasmusson I, et al: Mesenchymal stem cells for treatment of therapy-resistant graft-versus-host disease. Transplantation 81: 1390-1397, 2006.

14. Sun S, Guo Z, Xiao X, et al: Isolation of mouse marrow mesenchymal progenitors by a novel and reliable method. Stem Cells 21: 527-535, 2003.

15. Morris GP, Beck PL, Herridge MS, et al: Hapten-induced model of chronic inflammation and ulceration in the rat colon. Gastroenterology 96: 795-803, 1989.

16. Davaatseren M, Hwang JT, Park JH, et al: Poly- $\gamma$-glutamic acid attenuates angiogenesis and inflammation in experimental colitis. Mediators Inflamm 2013: 982383, 2013.

17. González R, Rodríguez S, Romay C, et al: Anti-inflammatory activity of phycocyanin extract in acetic acid-induced colitis in rats. Parmacol Res 39: 55-59, 1999.

18. Guo Y, Su L, Wu J, et al: Assessment of the green fluorescence protein labeling methods for tracking implanted mesenchymal stem cells. Cytotechnology 64: 391-401, 2012.

19. Yi T and Song SU: Immunomodulatory properties of mesenchymal stem cells and their therapeutic applications. Arch Pharm Res 35: 213-221, 2012.

20. Uccelli A, Moretta L and Pistoia V: Mesenchymal stem cells in health and disease. Nat Rev Immunol 8: 726-736, 2008.

21. Tolar J, Le Blanc K, Keating A and Blazar BR: Concise review: hitting the right spot with mesenchymal stromal cells. Stem Cells 28: 1446-1455, 2010.

22. Krampera M, Cosmi L, Angeli R, et al: Role for interferon-gamma in the immunomodulatory activity of human bone marrow mesenchymal stem cells. Stem Cells 24 : 386-398, 2006.

23. Tanaka F, Tominaga K, Ochi M, et al: Exogenous administration of mesenchymal stem cells ameliorates dextran sulfate sodium-induced colitis via anti-inflammatory action in damaged tissue in rats. Life Sci 83: 771-779, 2008.

24. Cooper HS, Murthy SN, Shah RS and Sedergran DJ: Clinicopathologic study of dextran sulfate sodium experimental murine colitis. Lab Invest 69: 238-249, 1993. 
25. Idriss HT and Naismith JH: TNF alpha and the TNF receptor superfamily: structure-function relationship(s). Microsc Res Tech 50: 184-195, 2000.

26. Locksley RM, Killeen $\mathrm{N}$ and Lenardo MJ: The TNF and TNF receptor superfamilies: integrating mammalian biology. Cell 104: 487-501, 2001.

27. Smith CA, Farrah T and Goodwin RG: The TNF receptor superfamily of cellular and viral proteins: activation, costimulation, and death. Cell 76: 959-962, 1994.

28. Ogata $\mathrm{H}$ and Hibi T: Cytokine and anti-cytokine therapies for inflammatory bowel disease. Curr Pharm Des 9: 1107-1113, 2003.

29. Zheng L, Fisher G, Miller RE, et al: Induction of apoptosis in mature T cells by tumour necrosis factor. Nature 377: 348-351, 1995.

30. Noti M, Corazza N, Mueller C, Berger B and Brunner T: TNF suppresses acute intestinal inflammation by inducing local glucocorticoid synthesis. J Exp Med 207: 1057-1066, 2010.

31. Olson TS, Reuter BK, Scott KG, et al: The primary defect in experimental ileitis originates from a nonhematopoietic source. J Exp Med 203: 541-552, 2006.

32. Reimund JM, Wittersheim C, Dumont S, et al: Mucosal inflammatory cytokine production by intestinal biopsies in patients with ulcerative colitis and Crohn's disease. J Clin Immunol 16: 144-150, 1996.

33. Armuzzi A, Lionetti P, Blandizzi C, Caporali R, et al: Anti-TNF agents as therapeutic choice in immune-mediated inflammatory diseases: focus on adalimumab. Int J Immunopathol Pharmacol 27 (1 Suppl): 11-32, 2014.
34. Chen FE and Ghosh G: Regulation of DNA binding by Rel/NF-kappaB transcription factors: structural views. Oncogene 18: 6845-6852, 1999.

35. Barnes PJ and Karin M: Nuclear factor-kappaB: a pivotal transcription factor in chronic inflammatory diseases. N Engl J Med 336: 1066-1071, 1997.

36. DiDonato JA, Hayakawa M, Rothwarf DM, Zandi E and Karin M: A cytokine-responsive IkappaB kinase that activates the transcription factor NF-kappaB. Nature 388: 548-554, 1997.

37. Schmitz ML, Bacher S and Kracht M: I kappa B-independent control of NF-kappa B activity by modulatory phosphorylations. Trends Biochem Sci 26: 186-190, 2001.

38. Pizarro TT and Cominelli F: Cytokine therapy for Crohn's disease: advances in translational research. Annu Rev Med 58:433-444, 2007.

39. Elson CO, Cong Y and Weaver CT: Monoclonal anti-interleukin 23 reverses active colitis in a T cell-mediated model in mice. Gastroenterol 132: 2359-2370, 2007.

40. Atreya R, Mudter J, Finotto S, et al: Blockade of interleukin 6 trans signaling suppresses T-cell resistance against apoptosis in chronic intestinal inflammation: evidence in crohn disease and experimental colitis in vivo. Nat Med 6: 583-588, 2000.

41. Suenaert P, Bulteel V, Lemmens L, et al: Anti-tumor necrosis factor treatment restores the gut barrier in Crohn's disease. Am J Gastroenterol 97: 2000-2004, 2002. 